\title{
Tétano acidental no Estado do Ceará, entre 2002 e 2005
}

\author{
Accidental tetanus in the State of Ceará, between 2002 and 2005
}

\author{
Alexsandra Rodrigues Feijão $0^{1}$, Daniele Mary Silva de Brito ${ }^{1}$, \\ Dalila Augusto Peres ${ }^{2}$ e Marli Teresinha Gimeniz Galvão ${ }^{3}$
}

\begin{abstract}
RESUMO
Descrevem-se as características demográficas e clínicas de indivíduos com tétano acidental no Ceará. Analisou-se, retrospectivamente, 131 casos de tétano acidental notificados em hospital de referência estadual entre 2002 e 2005. Destes, a maioria (84,8\%) ocorreu entre homens e em moradores da zona urbana (93,2\%). A faixa etária com maior incidência foi a de 35 a 49 anos (35,2\%). História vacinal completa esteve relatada em quatro (3\%) casos, sendo ferimento perfurocortante o mais observado, e os membros inferiores os mais acometidos. $O$ trismo esteve relacionado a $86,2 \%$ dos casos, seguido por contraturas (54,1\%). Entre os notificados bouve 95,5\% confirmados, cujo óbito incidiu em 33 (26,4\%) pacientes. Conclui-se que o tétano acidental no Ceará atinge a população de maneira expressiva, apesar de ser uma doença imunoprevinível. Urge autoridades e órgãos gestores da saúde programarem estratégias que visem mudanças de políticas públicas relacionadas à cobertura vacinal dos susceptiveis, principalmente adultos.
\end{abstract}

Palavras-chaves: Tétano acidental. Epidemiologia em saúde.

\section{ABSTRACT}

The demographic and clinical characteristics of individuals with accidental tetanus in Ceará are described. This was retrospective analysis of 131 cases of accidental tetanus notified in a state referral hospital between 2002 and 2005. Most of these cases (84.8\%) occurred among men and among people living in the urban zone (93.2\%). The age group with highest incidence was between 35 and 49 years old (35.2\%). A complete vaccination history was reported in four (3\%) cases. Puncturing/cutting wounds were the cause most commonly observed and the legs were the area most affected. Trismus (lockjaw) was reported in $86.2 \%$ of the cases, followed by muscle contractions (54.1\%). Among the notified cases, 95.5\% were confirmed and 33 patients (26.4\%) died. We conclude that accidental tetanus in Ceará significantly affects the population, in spite of being a disease that is preventable by immunization. The health authorities and health administration entities are urged to program strategies that aim towards changes in public policies concerning the vaccination coverage for susceptible individuals, particularly adults.

Key-words: Accidental tetanus. Health epidemiology.

Desde os tempos mais remotos, o tétano assola a humanidade e estima-se que acontecem cerca de 500 mil a um milhão de casos por ano no mundo, sobretudo nos países subdesenvolvidos. Em áreas de maior desenvolvimento socioeconômico e cultural, cuja população é vacinada sistematicamente, este é pouco freqüente ${ }^{8}$.

No Brasil, considera-se a região Nordeste um local onde a desigualdade socioeconômica sobrepuja um perfil diferenciado de adoecer e morrer da sua população, contribuindo de forma importante nas estatísticas nacionais. Nesta região, concentra-se cerca da metade dos casos de tétano neonatal e um terço do tétano acidental. Tais índices sugerem a necessidade de medidas mais efetivas de vigilância e controle constante da doença 9 .
Apesar dos investimentos para profilaxia do tétano, este continua presente em todo o território nacional. Como população mais acometida, sobressai a masculina, provavelmente em decorrência da sua exposição no trabalho. Portanto, o tétano constitui risco ocupacional entre trabalhadores, principalmente os da indústria da construção civil, da agricultura e da pecuária. Contudo, mesmo diante dos diferentes riscos advindos do cotidiano profissional, estes trabalhadores não estão incluídos nos programas regulares de imunização $0^{37}$, tornando-se as vítimas mais constantes do tétano acidental ${ }^{375}$.

0 presente estudo teve como objetivo descrever as características demográficas e clínicas dos pacientes com tétano acidental notificados e tratados em hospital especializado em Fortaleza, CE.

\footnotetext{
1. Hospital São José de Doenças Infecciosas, Fortaleza, CE. 2. Núcleo de Vigilância Epidemiológica, Hospital São José de Doenças Infecciosas, Fortaleza, CE. 3. Curso de Graduação e Pós-Graduação em Enfermagem da Universidade Federal do Ceará, Fortaleza, CE.

Endereço para correspondência: Dr ${ }^{a}$ Alexsandra Rodrigues Feijão. Gerência de Enfermagem/Hospital São José de Doenças Infecciosas. Rua Nestor Barbosa 315, Bairro Parquelândia, 60455-610 Fortaleza, CE.

Tel: 5585 3101-2337

e-mail alexsandrarf@hotmail.com

Recebido em: 05/01/2007

Aceito em: 12/07/2007
} 


\section{MATERIAL E MÉTODOS}

De acordo com o recomendado, a investigação cumpriu 0 estabelecido pelo Conselho Nacional de Ética em Pesquisa, e foi aprovada por Comitê de Ética em Pesquisa da Instituição lócus do estudo. Desenvolveu-se, então, pesquisa de caráter descritivo e retrospectivo no Hospital São José (HSJ) de Doenças Infecciosas de Fortaleza, CE, serviço de referência estadual para doenças infecto-contagiosas.

Para captação dos dados, utilizaram-se as informações das fichas de investigação epidemiológica compiladas no Sistema de Informação de Agravos de Notificação (SINAN) do Núcleo Hospitalar Epidemiologia do referido hospital, analisando-se 131 casos de tétano acidental notificados de janeiro de 2002 a dezembro de 2005. Em virtude de o HSJ ser o serviço de saúde que atendeu 95\% dos casos de tétano acidental ocorridos no Estado no período, neste estudo indicam-se os casos do Estado do Ceará.

Enquanto, o marco inicial da coleta de dados foi escolhido em decorrência da alteração da base de dados do SINAN ocorrida no ano anterior, sendo utilizada a partir deste período a versão atual, o final devido ao encerramento oportuno dos casos no momento da coleta de dados.

Como variáveis analisadas, incluíram-se as características demográficas e clínicas, classificação final e evolução dos casos. A compilação dos dados realizou-se por meio do software Tabwin ${ }^{\circledR}$, gerado a partir do SINAN, e a análise deu-se mediante freqüiência absoluta e relativa, cujos dados foram analisados de acordo com a literatura.

\section{RESULTADOS}

Nos quatro anos (2002 - 2005) avaliados, houve distribuição equivalente entre 2002 e 2004, e menor percentual em 2005. Do total avaliado, 84,8\% dos casos concentravam-se em indivíduos do sexo masculino e os demais no feminino. A maior parcela dos acometidos pelo tétano situa-se na faixa etária de 35 a 49 anos (35,2\%). Contudo, houve ocorrência em crianças com idade inferior a 10 anos $(3,8 \%)$, como mostra a Tabela 1 .

Quanto à escolaridade, 24,4\% dos pacientes possuíam no máximo o segundo grau e 20,6\% eram de analfabetos e parcela expressiva informou situação escolar ignorada (55\%).

Significativo número de casos ocorreu entre indivíduos da zona urbana (93,2\%). A grande maioria tinha profissão de agricultores, pedreiros e serviços de limpeza, no total de 40,3\% dos casos.

Em relação à história vacinal, apenas 3\% dos pacientes informaram vacinação antitetânica completa em períodos anteriores de suas vidas. Já, aqueles que não referiram vacinação prévia somaram 42,7\%. Entretanto, maior (47,3\%) parcela dos casos notificados constava informação ignorada na ficha de investigação do SINAN.
Tabela 1 - Caracterização dos 131 casos de tétano acidental notificados de 2002 a 2005.

\begin{tabular}{|c|c|c|}
\hline Variável & Número & Percentual \\
\hline \multicolumn{3}{|l|}{ Ano } \\
\hline 2002 & 33 & 25,1 \\
\hline 2003 & 39 & 29,8 \\
\hline 2004 & 35 & 26,8 \\
\hline 2005 & 24 & 18,3 \\
\hline \multicolumn{3}{|l|}{ Sexo } \\
\hline masculino & 111 & 84,8 \\
\hline feminino & 20 & 15,2 \\
\hline \multicolumn{3}{|l|}{ Faixa etária (anos)* } \\
\hline$\leq 9$ & 5 & 3,8 \\
\hline $10-19$ & 4 & 3,0 \\
\hline $20-34$ & 23 & 17,5 \\
\hline $35-49$ & 46 & 35,2 \\
\hline $50-64$ & 28 & 21,4 \\
\hline$\geq 65$ & 25 & 19,1 \\
\hline \multicolumn{3}{|l|}{ Procedência } \\
\hline zona urbana & 122 & 93,2 \\
\hline zona rural & 9 & 6,8 \\
\hline \multicolumn{3}{|l|}{ Escolaridade } \\
\hline primeiro ou segundo grau & 32 & 24,4 \\
\hline analfabeto & 27 & 20,6 \\
\hline ignorado & 72 & 55,0 \\
\hline \multicolumn{3}{|l|}{ Ocupação } \\
\hline agricultor & 24 & 18,3 \\
\hline pedreiro & 18 & 13,7 \\
\hline serviços de limpeza & 11 & 8,3 \\
\hline comerciante/vendedor & 6 & 4,5 \\
\hline dona de casa ${ }^{* *}$ & 9 & 6,8 \\
\hline aposentado & 7 & 5,3 \\
\hline estudante & 5 & 3,8 \\
\hline outros & 51 & 39,0 \\
\hline \multicolumn{3}{|l|}{ História de vacinação } \\
\hline esquema completo & 4 & 3,0 \\
\hline esquema incompleto & 9 & 6,8 \\
\hline ignorado & 62 & 47,3 \\
\hline nunca vacinado & 56 & 42,7 \\
\hline
\end{tabular}

* faixas etárias sugeridas pelo programa

*** refere-se, apenas, às mulheres cuja ocupação era dona de casa

A Tabela 2 mostra os aspectos clínicos da doença. Quanto ao tipo de ferimento, 40,5\% aconteceram por perfuração, seguido por escoriação e laceração. Ressalta-se que também houve casos após procedimentos cirúrgicos (10\%), após acidentes por queimaduras $(1,5 \%)$ e em outros tipos de ferimentos $(10 \%)$.

Quanto à região afetada pelo ferimento, os membros inferiores $(57,3 \%)$ foram os mais acometidos, e os pés responderam por $77,3 \%$ de exposição dessas lesões (dados não apresentados). Em três casos, a cavidade oral foi a região do corpo que apresentou porta de entrada para o Clostridium tetani, situação pouco divulgada em estudos.

Como evidenciado, entre os sinais característicos da doença, o trismo $(86,2 \%)$, as contraturas $(54,1 \%)$ e a rigidez de 
Tabela 2 - Distribuição dos 131 casos de tétano acidental notificados entre 2002 e 2005, de acordo com quadro clínico.

\begin{tabular}{lcc}
\hline Variável & Número & Percentual \\
\hline Tipo de ferimento* & & \\
perfuração & 53 & 40,5 \\
escoriação & 28 & 21,3 \\
laceração & 22 & 16,7 \\
cirúrgico & 13 & 10,0 \\
queimadura & 2 & 1,5 \\
$\quad$ outros & 20 & 10,0 \\
Região afetada pelo ferimento & & \\
membros inferiores & 75 & 57,3 \\
membros superiores & 17 & 13,0 \\
cabeça & 10 & 7,6 \\
tronco & 4 & 3,0 \\
cavidade oral & 3 & 2,3 \\
outras & 22 & 16,8 \\
Sinais e sintomas* & & \\
trismo & 113 & 86,2 \\
contraturas & 71 & 54,1 \\
rigidez de nuca & 68 & 52,0 \\
rigidez abdominal & 23 & 17,5 \\
rigidez de membros & 15 & 11,4 \\
opistótomo & 14 & 10,6 \\
riso sardônico & 11 & 8,3 \\
\hline
\end{tabular}

* um paciente pode ter apresentado concomitantemente um ou mais tipos de ferimento e sinais e sintomas

nuca (52\%) constituíram os principais sinais, associados ou não uns aos outros.

Em relação à classificação final, dos 131 casos notificados como tétano, entre eles, seis $(4,5 \%)$ casos notificados foram descartados, sendo três casos de trismo por processo inflamatório e outros três por tetania decorrentes de outras causas. Por sua vez, dos 125 confirmados como tétano acidental, houve substancial ocorrência de óbitos, com letalidade de 26,4\% (Tabela 3).

Tabela 3 - Classificação final dos 131 casos notificados como tétano acidental entre 2002 e 2005.

\begin{tabular}{lrr}
\hline Classificação final dos casos & Número & Percentual \\
\hline Tétano confirmado & 92 & \\
$\quad$ cura & 33 & \\
$\quad$ óbito & 125 & 95,5 \\
$\quad$ subtotal & & \\
Tétano descartado & 3 & \\
$\quad$ trismo por processo inflamatório & 3 & \\
tetania por outras causas & 6 & 4,5 \\
$\quad$ subtotal & 131 & 100,0 \\
\hline Total & & \\
\hline
\end{tabular}

\section{DISCUSSÃO}

Mesmo com a profilaxia contra o tétano disponível gratuitamente, nos serviços de saúde pública do país, observa-se a ocorrência da doença em todas as regiões. No Ceará, o tétano acidental aconteceu de maneira semelhante durante os anos avaliados. Entretanto, outros estados da região Sudeste e Sul demonstram declínio da sua ocorrência, a exemplo de São Paulo ${ }^{2}$ e Santa Catarina ${ }^{10}$.

Segundo divulgado, o sexo masculino é o grupo mais atingido, pois não existe campanha de vacinação direcionada a estes indivíduos na fase adulta. Já para as mulheres, há estratégia vacinal, direcionada à prevenção do tétano neonatal, durante 0 período gestacional, para a proteção do recém-nascido.

0 elevado número de casos em indivíduos acima de 50 anos deve-se ao fato de que o envelhecimento, devido a sua fisiologia, torna as pessoas mais susceptíveis a acidentes, ocasionados por instrumentais e por quedas, havendo redução dos reflexos, da acuidade visual e auditiva e da habilidade motora ${ }^{7}$.

Outro aspecto a se destacar, foi a presença de casos em menores de 10 anos, devido ao esquema vacinal incompleto associado e à relação do tétano acidental com atividades de lazer $^{4}$. Tal fato indica a necessidade de se intensificar medidas de prevenção em ambos os sexos e nas diferentes faixas etárias da população do estado.

De acordo com o Ministério da Saúde, a medida de controle para o tétano acidental é a manutenção de cobertura vacinal adequada e, especificamente, em crianças, gestantes e adultos da terceira idade. Indica-se ainda para indivíduos com presença de úlcera crônica de pernas, mal perfurante plantar e trabalhadores de risco, tais como agricultores e operários da construção civil ${ }^{4}$. No entanto, vários fatores influenciam a inadequada cobertura vacinal nestes grupos prioritários, entre eles, aspectos individuais, culturais, sociais, econômicos, e de acessibilidade aos serviços de saúde.

Segundo destacado por pesquisadores, a vacinação de adultos é mais complexa, pois a maioria não procura regularmente serviços de saúde, além de existirem oportunidades perdidas, como medidas associadas às ações nos programas de doenças crônicas, de controle do câncer cérvico-uterino, e consultas em serviços de urgência ou de pronto-atendimento ${ }^{2}$.

No âmbito de pesquisas, o padrão mais característico da ocorrência do tétano acidental tem sido em indivíduos da zona rural. Contudo, acidentes ocorridos na zona urbana prevaleceram no Estado de Santa Catarina ${ }^{10}$ e no Ceará, evidenciados por índice elevado entre os casos estudados.

Quanto à ocupação, os indivíduos mais acometidos foram os agricultores, pedreiros e indivíduos de serventia de limpeza. Esses resultados revelam a relação direta entre o adoecimento e o risco ocupacional a que estas pessoas estão expostas.

No Brasil, as áreas urbana, suburbana e rural são definidas por leis municipais. Consideram-se populações urbanas e suburbanas aquelas residentes nas áreas das cidades (sedes municipais), das vilas (sedes distritais) e também das aglomerações definidas como urbanas por órgão oficiais ${ }^{8}$. Isto implica certa relativização dos conceitos de procedência urbana e rural, justificando a relação, aparentemente discordante, demonstrada neste estudo entre ocupação mais acometida (agricultores) e procedência da maioria dos casos (urbana).

Verifica-se ainda que parcela significativa dos casos encontrase em situações ocupacionais não relacionadas ao risco direto 
da aquisição do esporo do bacilo. Ressalta-se que as mulheres (donas de casa) se não estiverem em idade fértil, ficam fora da faixa etária priorizada pelas ações de assistência pré-natal e, portanto, susceptíveis ao tétano acidental.

Para controle do tétano acidental, define-se como pessoa adequadamente vacinada aquela que tomou três doses de toxóide tetânico (DPT, DT, dT, TT), tendo sido a última dose há menos de dez anos ${ }^{4}$. Segundo evidenciado, grande (42,7\%) parte dos pacientes não foram imunizados. Isto mostra a deficiência de medidas preventivas.

É importante salientar elevado (47,3\%) percentual dos casos com o preenchimento da informação ignorada no campo destinado à situação vacinal. Ante a circunstância, inferem-se alguns motivos da inadequação do preenchimento da ficha de investigação do SINAN, tais como o desconhecimento da situação vacinal e a inobservância ou não relevância da informação atribuída ao preenchimento realizado pelo investigador.

Com vistas à determinação dos fatores preditivos para o diagnóstico, é necessária a avaliação criteriosa de fatores relacionados à aquisição do bacilo, como tipo elocal do ferimento, sinais e sintomas, provável fonte de infecção e a situação vacinal. É oportuno lembrar que quanto maior o período de incubação, pior o prognóstico do paciente. Em qualquer situação, exige-se adequada assistência aos ferimentos ${ }^{2}$.

Dentre os casos estudados, observa-se que a maior parcela se deu em decorrência de ferimento perfurante. Também foram altas as taxas de ferimento por escoriação, laceração e outros tipos. Esses dados são semelhantes aos observados na região sul do país ${ }^{10}$.

Salienta-se que qualquer solução de continuidade com presença de tecido necrosado, contaminado com terra, poeira e fezes, favorece a colonização da bactéria após a deposição dos esporos. Isso justifica a ocorrência de casos decorrentes de queimaduras, feridas cirúrgicas, aborto infectado, cárie dentária e ulcera de pernas ${ }^{4}$.

Ao associar-se tipo de ferimento com a variável ocupação, percebe-se que estes acidentes talvez tenham acontecido durante o trabalho, em virtude dos instrumentos utilizados. Conforme observado, os pés foram os mais atingidos, provavelmente, também, como resultado do hábito de andar e trabalhar descalço, situação evitável pelo uso de calçados adequados às práticas profissionais, possibilitando a redução da doença.

Neste estudo, a evidência do trismo foi sinal predominante entre os casos avaliados de tétano acidental. Situação semelhante também foi observada no interior de São Paulo ${ }^{2}$, em investigaçõos nas quais a presença conjugada de contraturas ${ }^{1}$ e rigidez de nuca ${ }^{16}$ demonstraram associação significativa com a de morte por tétano.

Quanto aos casos descartados, estes englobam dois diagnósticos diferenciais do tétano acidental: 0 trismo por processos inflamatórios locais associados a infecções dentárias, amigdalite, artrite temporomandibular, patologias do ouvido médio, e a tetania por outras causas como hipocalemia, magnesemia, doença do soro, intoxicações exógenas, histeria, menigoencefalite, peritonite e raiva humana ${ }^{4}$.
Para determinação da letalidade do tétano acidental considerou-se a razão entre os óbitos ocorridos em relação aos casos confirmados. Assim neste estudo, a letalidade chegou a $26,4 \%$. No entanto, a evolução clínica dos pacientes não diferiu da relatada na literatura, cuja ocorrência de óbitos foi semelhante no interior paulista ${ }^{4}$ e inferior às taxas encontradas na Bahia ${ }^{9}$. Embora a cura tenha se verificado em $73,6 \%$ dos casos considera-se baixa, pois o tétano é doença imunoprevenível.

Devido a diversas complicações, o tratamento do tétano acidental requer na maioria das vezes leitos em Unidade de Terapia Intensiva (UTI) ${ }^{9}$. Como se sabe, o custo para manutenção de um dia de internação em UTI nos serviços de saúde é elevado e, à época, apresentava valor médio de $\mathbf{R} \$ 1.138,00$, enquanto uma dose de vacina antitetânica tinha-se um valor aproximado de $\mathrm{R} \$ 0,10$ (valores de custos referidos pela direção administrativa do HSJ em comunicação pessoal, 2005). Ante o fato, depreendese que efetivamente 0 custo da vacinação antitetânica para a população geral é pequena e o beneficio social e econômico gerado com a prevenção do tétano acidental é indiscutível.

Neste contexto, os profissionais de saúde devem promover ações de mobilização social e traçar estratégias nos serviços de saúde para facilitar o acesso da comunidade e programar ações voltadas para imunoprofilaxia do tétano, independente de sexo, idade, ocupação ou zona de moradia.

Sugere-se ao estado disponibilizar a vacina antitetânica, mediante promoção de campanhas anuais com enfoque direcionado ao homem para a prevenção do tétano. Citam-se, ainda, como estratégias plausíveis o oferecimento da vacinação nos programas de doenças crônicas (hipertensão e diabetes), e durante 0 controle de câncer ginecológico, além de outras ações de saúde mais permanentes. Destaca-se também estratégia para ampliação da cobertura vacinal o oferecimento da imunização quando da emissão de documentos pessoais a exemplo da expedição do título de eleitor e da carteira de habilitação de condutor de veículos.

Associada a essas sugestões, para a prevenção do tétano acidental, ressalta-se a atuação dos profissionais de saúde para um diagnóstico mais precoce. Desta forma, pode-se atenuar os casos que culminam em óbitos.

\section{REFERÊNCIAS}

1. Greco JB, Tavares-Neto J, Greco Jr J. Accidental tetanus: prognosis evaluation in a historical series at a hospital in Salvador, Bahia, Brasil. Revista do Instituto de Medicina Tropical de São Paulo 45:35-40, 2003.

2. Lima VMSF, Garcia MT, Resende MR, Nouer SA, Campos E0, Papaiordanou PMO, Silva LJ. Tétano acidental: análise do perfil clínico e epidemiológico de casos internados em hospital universitário. Revista de Saúde Pública 32:166-171, 1998.

3. Melo HRL, Brito CAA, Miranda Filho DB, Sousa SG, Henriques APC, Silva OB. Condutas em doenças infecciosas. Medsi Editora Médica e Científica Ltda, Rio de Janeiro, 2004.

4. Ministério da Saúde. Casos de agravos e doenças infecciosas e parasitárias notificadas de janeiro a dezembro de 1997 e igual período de 1998, por Unidade Federada, Brasil. Informe Epidemiológico do Sistema Único de Saúde 7:117-120, 1998 . 
5. Ministério da Saúde. Guia Brasileiro de Vigilância Epidemiológica. Brasília, p. 421-426, 1998

6. Miranda-Filho DB, Ximenes RAA, Bernardino SN, Escarião AG. Identification of risk factors for death from tetanus in Pernambuco, Brazil: a case-control study. Revista do Instituto de Medicina Tropical de São Paulo 42:333-339, 2000.

7. Pagliuca LMF, Feitoza AR, Feijão AR. Tétano na população geriátrica: problemática da Saúde Coletiva? Revista Latino-Americana de Enfermagem 9:69-75, 2001.
8. Rouquairol MZ, Almeida Filho N. Epidemiologia e Saúde. 6ª edição, Medsi Editora Médica e Científica Ltda, Rio de Janeiro, 2003.

9. Sistema Único de Saúde. Banco de dados DATASUS. Série histórica de casos de doença de notificação compulsória por unidade federada (1980-2003) http://portalweb02. saude.gov.br/portal/sus/visulizar_texto.cfm? Acesso em 07 out 2005.

10. Viertel IL, Amorin L, Piazza U. Tétano acidental no estado de Santa Catarina, Brasil: aspectos epidemiológicos. Epidemiologia dos Serviços de Saúde 14:33-40, 2005. 